\title{
The Effects of Tactile Feedback and Movement Alteration on Interaction and Awareness with Digital Embodiments
}

\author{
Andre Doucette ${ }^{1}$, Regan L. Mandryk ${ }^{1}$, Carl Gutwin ${ }^{1}$, Miguel Nacenta ${ }^{2}$, Andriy Pavlovych ${ }^{1}$ \\ ${ }^{1}$ Department of Computer Science \\ University of Saskatchewan, \\ Saskatoon, Canada \\ ${ }^{2}$ School of Computer Science, \\ University of St Andrews, \\ St Andrews, UK \\ \{firstname.lastname\}@usask.ca, mans@st-andrews.ac.uk, andriyp@gmail.com
}

\begin{abstract}
Collaborative tabletop systems can employ direct touch, where people's real arms and hands manipulate objects, or indirect input, where people are represented on the table with digital embodiments. The input type and the resulting embodiment dramatically influence tabletop interaction: in particular, the touch avoidance that naturally governs people's touching and crossing behavior with physical arms is lost with digital embodiments. One result of this loss is that people are less aware of each others' arms, and less able to coordinate actions and protect personal territories. To determine whether there are strategies that can influence group interaction on shared digital tabletops, we studied augmented digital arm embodiments that provide tactile feedback or movement alterations when people touched or crossed arms. The study showed that both augmentation types changed people's behavior (people crossed less than half as often) and also changed their perception (people felt more aware of the other person's arm, and felt more awkward when touching). This work shows how groupware designers can influence people's interaction, awareness, and coordination abilities when physical constraints are absent.
\end{abstract}

\section{Author Keywords}

Embodiments, tabletop groupware, awareness, coordination.

\section{ACM Classification Keywords}

H.5.M. Information interfaces (e.g., HCI): Miscellaneous.

\section{General Terms}

Human Factors; Design; Measurement.

\section{INTRODUCTION}

Digital tables provide large workspaces where people can share and manipulate computational artifacts. Digital tables are natural sites for collaboration: they allow rich verbal and non-verbal communication, and they let people use well-practiced coordination mechanisms from everyday experience with physical tables and surfaces [22,25,32].

(C) ACM 2013. This is the author's version of the work. It is posted here for your personal use. Not for redistribution. The definitive Version of Record was published in the Proceedings of the SIGCHI Conference on Human Factors in Computing Systems (CHI '13),

http://dx.doi.org/10.1145/2470654.2466250

The copy of record of the paper can be found in:

http://dl.acm.org/citation.cfm?doid=2470654.2466250
The shift from physical to digital environments, however, can also change the way that people interact with objects and with each other. Designers of tabletop groupware systems must take these changes into consideration to effectively support group work - but little is currently known about how the move from physical to digital can affect behavior. One issue that has strong ramifications for groupware design is how the table's input technique, and the embodiment type that results from that input, affects coordination and awareness in tabletop activity.

Digital table systems can employ two main types of input: direct touch, in which people use their real arms and hands to manipulate objects on the table, or indirect input, in which people use an input device like a mouse, and where people are represented on the table through a virtual embodiment such as a pointer, a line, or an arm drawn on the surface. Indirect input is valuable (and sometimes the only option) when tables are large and items out of people's physical reach require them to move around the table [31].

The type of input, and the resulting embodiment type, can dramatically influence tabletop interaction. Recent research shows that one basic interaction - touching or crossing another person's arm - is very rare with direct touch and physical arms, but is common with indirect input and virtual arms [8]. This difference is not just a curiosity, because the strong avoidance of touching and crossing with physical arms is one of the awareness mechanisms that helps people understand and manage shared access to public space. Touch avoidance is evident in several complex behaviors in tabletop work: for example, it plays a role in people's fine-grained awareness of others' locations, in dynamic negotiation of access to shared objects, in people's ability to protect areas of the workspace, and in accommodation behavior, where people move out of the way when someone needs to reach past them [8].

When tables use indirect input instead of direct touch, this strong mechanism underlying awareness and coordination disappears from the environment, leading to dramatically different crossing and touching behavior with virtual arm embodiments [8]. Although this loss of a constraint can be useful in some situations, in others it can cause interaction problems: people are less aware of others, less able to avoid access conflicts, and less able to protect objects and maintain control over their personal work territory [8]. 
Designers of tabletop groupware systems need to better understand the factors that govern and shape interaction behavior over tables - not to replace touch avoidance, and not to simply replicate the physical world, but to determine whether some of the valuable aspects of physical interaction can be added to the designer's toolbox. It is not immediately clear what these factors might be; for example, an earlier study showed that increasing the visual fidelity of an arm embodiment did not reduce people's crossing and touching behavior [8]. However, this study also suggested two factors for further study: tactile feedback, which is one of the foundations of touch avoidance in the physical world; and movement alteration, which can prescribe the difficulty of interacting in the same table space as another person.

In this paper, we investigate embodiment augmentations through a tabletop study designed to test the effects of tactile feedback and movement alterations on group reaching behavior and awareness. To test tactile feedback, we attached vibration outputs to either the participant's mouse or to their thigh. To test movement alteration, we changed the cursor's movement speed when embodiments touched (either slowing it or stopping it altogether). Participants carried out tabletop tasks with all augmentations types, as well as a control condition with no augmentations. Our study provides three main results:

- Both augmentation types significantly changed tabletop behavior: tactile feedback reduced crossings by as much as one-half; movement alterations reduced crossings by as much as $75 \%$;

- Both augmentation types also significantly changed people's feelings of awareness, awkwardness, and intrusion; augmentations were rated significantly higher than the control; the ratings for augmentations are similar to those reported for physical arms [8].

- Participants reported that the addition of tactile feedback and movement alteration was more annoying to use than an un-augmented embodiment; they also reported that having to coordinate was not very frustrating overall.

Our study is the first investigation of using embodiment augmentations to shape people's collaborative interactions over digital tables, and the first to show how tactile feedback and movement alteration can modulate people's behaviour in co-located collaborative situations. Our research provides a first step towards a richer set of design capabilities for designers of tabletop groupware to enable a broader range of group tabletop applications.

\section{RELATED WORK}

Our work draws from previous research into physical touch, personal space in the physical and digital worlds, tabletop embodiment and input, and access control to shared items.

\section{Physical Touch}

Touch is the most intimate interpersonal communication channel [37]. Work in body-accessibility shows that the location of a touch, and the intimacy of the touched area, are central to people's comfort level in being touched [19]. Hands and arms are the least intimate touch locations, and the thighs are one of the most intimate and guarded $[14,19,26]$. The social rules of touch, including who can do the touching [14], manifest themselves in the well-studied phenomenon of touch avoidance [1]. Touch avoidance research focuses on the circumstances that cause people to avoid tactile contact with each other. People's natural ability and inclination to avoid touching others is particularly prominent during tabletop work, where people avoid crossing over or under another person's arm; instead, people take turns interacting in the workspace [8]. Previous work showed that touch avoidance does not transfer when physical arms are replaced by digital embodiments [8].

HCI researchers have studied several aspects of touch that are peripherally related to our research. For example, research into mediated social touch attempts to support touch over a distance through tactile or kinesthetic feedback (see [12] for a review). Other researchers created haptic or tactile feedback systems for a variety of purposes, ranging from increasing presence [28], expressing and interpreting emotion [3], providing spatial information to blind users [29], and encouraging users to take breaks [7].

\section{Personal Space in Digital Environments}

People vary in their willingness and comfort letting others into the space surrounding them (i.e., personal space) [13]. Work on VR suggests that personal space also applies to digital avatars - researchers showed that invasions of avatar personal space make people uncomfortable $[18,33,34]$. People also avoid making others uncomfortable (e.g., by not walking through their avatar) [33].

There is little previous work investigating personal space in tabletop or other groupware systems. Previous researchers assumed that social protocols would be enough to guide users' behaviour (e.g., [9]). Other researchers reported that users reached through each other's personal workspaces, even stealing words from others [23], suggesting that the digital world does not have the same social protocols as the physical world. For example, in a remote task, people had little issue sitting "in each others' laps" [35].

Territoriality research [32] showed that people partition tabletop workspaces into personal and public workspaces. Personal workspaces are often directly in front of each user, simulating a version of personal space. Some evidence suggests that indirect input affects natural collaborative behaviours such as territoriality [32], and leads to an increase in coordination problems [24].

\section{Tabletop Embodiments}

Embodiments represent users in the workspace. They allow users to interact with the workspace, and allow others to track a user's actions. Previous work in CSCW has studied several kinds of embodiments, such as avatars, telepointers, and video embodiments. 
Tabletop embodiments can be either physical (people use their arms and hands to interact in the workspace) or digital (a visual representation of the user, with a form of indirect input, like a mouse). There are many advantages of digital embodiments over physical embodiments (e.g., ability to reach [24]). Tabletop embodiments can be cursors, pantographs (a line connecting cursor and user), arm embodiments (pantographs with more "arm-like" visuals) [31], or video of physical arms for distributed tables [36].

The choice of embodiment can affect several aspects of group interaction. Prior research in this area focused on comparing direct and indirect input and the effects on performance [11], coordination and conflict [15,25,31], and spatial interference [36,39].

\section{Coordination and Access Control in Groupware}

Access to shared resources is an important issue for groupware designers. Early groupware researchers examined role-based access and distributed-systems approaches such as locking and serialization [9]. Recently, researchers have started investigating different possible techniques to enable collaboration, and how these interact with social protocols and affect behaviour [22,23,38].

Researchers identified that social protocols were often enough to support coordination and turn taking without needing more explicit access control, as long as there was adequate awareness information about others' locations and activities [9]. What happens to social protocols when moving from physical to digital is still not well understood, with some researchers suggesting that physical protocols do not transfer directly to digital [17,23]. Only a few researchers have investigated adding dynamic rather than role-based or explicit access control; for example, [30] investigated how competitive behaviour in a game was affected by rules and policies that control who can manipulate which objects and when. An alternative approach to access control is to introduce a cost to the behaviour. Previous researchers have shown that there are benefits to making a task more difficult to complete. For example, performance costs can aid spatial learning [6] and can improve planning on a task without impairing the final result [27]; and adding visual difficulties can induce deeper learning strategies in information visualizations [16].

To our knowledge, there has not been any substantial study addressing the relationship between interpersonal physical touch, coordination, and how digital environments can replicate or substitute this fundamental proxemic behaviour.

\section{DIGITAL TABLETOP STUDY}

People avoid touching and crossing physical arms when working at a table. In many situations (e.g., when working remotely, or on large tables), indirect input is more appropriate; however, previous work has shown that people have little issue crossing digital embodiments, regardless of the visual design [8]. The lack of awkwardness of touching embodiments means that people may not maintain awareness of others' embodiment locations and actions, in stark contrast to the continuous, rich, and up-to-date information people collect of others' physical arms.

We introduce two augmentations to affect interaction (i.e., embodiment crossing) and to increase awareness in colocated tabletop collaboration. Our augmentation types are designed to bring attention to crossing behaviour through various levels of feedback. With physical arms, this feedback comes naturally through the social awkwardness of touching another person $[1,13]$ and also through the time it takes to reach around another person's arm. This suggests two styles of embodiment augmentations: introducing awkwardness and affecting movement.

\section{Embodiment Augmentations}

Based on our observations of physical arms in multiple pilot studies, we created two embodiment augmentation types: tactile feedback and movement alteration. Within these two types, we created both low and high levels.

\begin{tabular}{|c|c|c|}
\cline { 2 - 3 } \multicolumn{1}{c|}{} & Low & High \\
\hline Tactile Feedback & Mouse Vibration & Pocket Vibration \\
\hline Movement Alteration & Slowed Interaction & Blocked Interaction \\
\hline \multicolumn{2}{|c|}{ Table 1. Embodiment augmentation types. }
\end{tabular}

- In Mouse Vibration, a small vibrotactile motor buzzes inside of a custom-built mouse.

- In Pocket Vibration, a small vibrating box is placed on the front of each user's thigh.

- In Slowed Interaction, a control-display (C/D) gain decrease slows both embodiments when they cross.

- In Blocked Interaction, the embodiments cannot cross.

The tactile feedback replicates the social awkwardness of touching arms in the digital domain. Mouse vibration is the lower level of feedback because it is applied to a device that is held in the hand - the location considered least awkward to be touched [26]. The pocket places the feedback on the thigh, one of the most awkward locations to be touched [26]. Although participants are aware that they are not "touching" their partner, we believe control over producing vibration by touching embodiments would follow the same pattern of awkwardness from physical touching.

The movement alteration approach introduces feedback through performance by affecting people's ability to work quickly. The slowed interaction is the lower level because it only delays interaction using a C/D gain, creating a feeling of stickiness [21]. The blocked level is the high level because it prevents interaction (implemented through setting the $\mathrm{C} / \mathrm{D}$ gain to zero - as the cursor moves, there is no corresponding movement of the embodiment).

\section{Implementation of Augmentations}

The mice and pocket vibrating boxes each contained two cylindrical button-type vibrators, either wrapped in polymer foam and formed into a sturdy package using hot glue and 
adhesive tape (for the pocket devices), or placed into a desktop mouse. The individual motors inside each device were wired in parallel and were computer-controlled via a Phidgets analog board. As the board outputs only $20 \mathrm{~mA}$ per channel, and the motors required about $300 \mathrm{~mA}$, we added a transistor stage to each channel as a voltage buffer (also known as an emitter follower). The setup allowed us to reliably control the vibration strength of the devices. The vibrators ran continuously at the same frequency (approximately $150 \mathrm{~Hz}$, according to the manufacturer's specs) while embodiments were crossed.

The $\mathrm{C} / \mathrm{D}$ gain slowdown for slowed interaction was determined through pilot studies. We scaled all mouse movements down by a factor of 17 - a mid-level range that still allows movement, but is "sticky" enough to be noticeable. As noted above, to prevent embodiments from crossing, the factor for blocked was set to infinity.

\section{Study Setup}

To investigate how groups would respond to the augmented embodiments, we asked dyads to create six sets of individual haikus using a digital tabletop system.

\section{System and Task Descriptions}

We replicated the system and task used in [8]. Dyads were asked to build six individual haiku sets by dragging words from the shared center part of the table to their haiku paper in front of them (see Figure 1), where they assembled their haikus. The digital haiku papers measured $400 \times 175$ pixels. The digital words were large enough to be easily read by both participants from their seated location.

Dyads used a $125 \mathrm{~cm}$ x $88 \mathrm{~cm}$ top-projected tabletop system, with resolution of $1280 \times 960$. People sat side by side, and interacted with the tabletop with their mouse to the right of their digital haiku papers. It is common for pairs to sit sideby-side at real-world tables. This arrangement is also necessary when working with textual artifacts, given how much easier it is to read text oriented towards you. In addition, previous work showed that people associate orientation with ownership [20]. Our setup ensured people felt they could use any of the shared words on the table.

The attributes of the haiku building task are common in real world tasks. First, the tabletop is split into territories, common in tabletop work [32]. Second, the haiku task is a mixed-focus collaborative task [10], where users switch between individual and group work. The coordinated access to the shared words in the public space is the group work.

The six haiku building tasks used a different set of words from six topic pairs: Planet/Horse, Clothing/Book, Coffee/Cat, Car/Tree, Student/Dog, Lake/Chair. Topics were paired so that words from one topic were less useful to the other topic (e.g., 'flower' is more useful for a tree haiku than a car haiku). There were 174 shared words available for each haiku task: 36 words from each topic, plus 102 joiner words (e.g., 'the', 'and', 'of').
We arranged the words such that the words for each topic were on the opposite side of the table; for example, the 'tree' words were on the opposite side of the table to the 'tree' haiku (see Figure 1). This distribution encouraged people to reach to the other side of the table, increasing the chance of an arm crossing. We randomly distributed the words on the appropriate sides of the

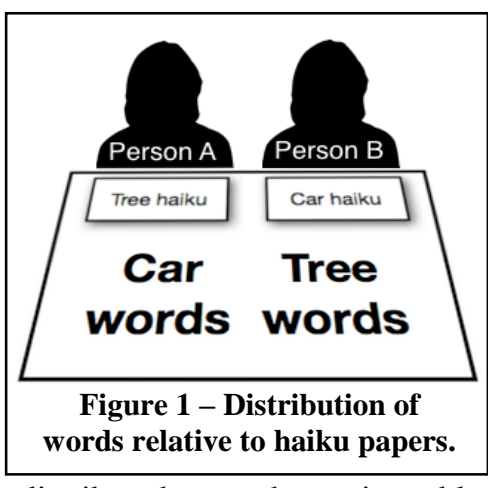
table, with joiner words distributed over the entire table. The distribution of words was stored such that each group saw the same words in the same location. Groups were told they could use any word in the shared space.

\section{Procedure}

During pilot testing, we found that groups quickly learned how to use the system to build their haikus, so no explicit training was required.

Participants completed the task using a baseline (unaugmented) embodiment, and the four augmented embodiments. The visual embodiment was based on the Transparent embodiment from [8], which had the best balance between interruption and noticeability. It is shaped like a real arm, and has $70 \%$ opacity. All embodiments were controlled with the mouse; the tip of the embodiment corresponded to the cursor location, and the embodiment created a straight line between the cursor location and the right side of the participant's haiku paper.

Participants all started the experiment by completing one haiku set using the baseline (non-augmented) embodiment. Following this baseline trial, participants were informed that they would receive feedback when they crossed their arms. A blank screen with the embodiments appeared, and groups were instructed to cross once to experience the feedback. The next haiku task was started immediately after, because we wanted to see how groups would adapt to the feedback and did not want all the adaption to happen in the training stage, where we were not logging interactions.

Augmented embodiments were presented in four orders to balance potential effects of presentation order. Half of the participants started with the tactile feedback augmentations and half with the movement alteration augmentations. Within those groups, the initial condition was balanced between the high and low levels. The order of the last two augmentations mirrored the order of the first two. Haiku topic pairs were presented in a single order.

We were interested in whether changes to behaviour from augmented embodiments lasts after the augmentation is removed. To investigate this 'permanence', we included a second baseline condition following the first augmentation. 
This allowed us to compare augmentation types in a withinsubjects design and compare the effect of permanence in a between-subjects design. Thus, the four orders were:

Baseline1-Blocked-Baseline2-Slowed-Pocket-Mouse Baseline1-Slowed-Baseline2-Blocked-Mouse-Pocket Baseline1-Mouse-Baseline2-Pocket-Slowed-Blocked Baseline1-Pocket-Baseline2-Mouse-Blocked-Slowed

Following the six haikus, dyads completed a postexperiment questionnaire to collect subjective responses. To ensure we did not bias participants into thinking about personal space and awkwardness, participants completed the questionnaire only after the last haiku.

\section{Participants}

Participants were asked to bring a friend or co-worker for the study. Users of tabletops in real work settings will likely work mostly with co-workers whom they know. We focus our research on work settings, so we tested this type of dyad. The median length of relationship for dyads was 30 months ( 2 months to 17 years). Ten dyads reported being friends, and six were class- or lab-mates. Dyads reported they interacted on average 3.75 times per week.

There were 32 participants (15 female, mean age 26.4). None participated in previous haiku-building studies. Twelve participants had never heard of digital tables; 12 had heard of them but never used one; and 8 had used a digital table before. Ten participants reported a Chinese language as their first language; 9 reported English; 5 dyads had other first languages.

Because people brought a partner, we did not control the distribution of sex in our dyads; yet, this balanced with 5 male-female, 6 male-male and 5 female-female dyads.

\section{Measures}

The system recorded the number of times people crossed embodiments. The number of crossings relates to people's willingness to touch each other. For Blocked, the system restricts people's ability to cross. Because our measure (crossings) is also the manipulation (i.e., we do not allow crossings), we only record new crossing events if people are blocked, move 50 pixels away (half the width of the embodiment), and then try to cross again.

Following the experiment we collected subjective responses to 7-point Likert scale questions about participants' awareness of their partner's embodiment, their feelings of awkwardness reaching and crossing, and their feelings of invasion of personal space. In addition, we also had a semistructured interview with dyads following the session.

\section{Data Analyses}

Visual inspection of the distribution of crossing counts indicate that parametric analyses were adequate; therefore we ran RM-ANOVAs with $\alpha=0.05$. Crossings are difficult to attribute to one or the other participant, so we report these per dyad. We determined that order of presentation of augmentations had no effect on the number of crossings through a RM-ANOVA with order as a between-subjects factor. There was no main effect of order on the number of crossings $\left(F_{(3,12)}=0.3, p=0.369, \eta^{2}=0.07\right)$, and no interaction of order with augmentation type $\left(F_{(12,48)}=1.1, p=0.368\right.$, $\eta^{2}=0.22$ ). Subsequent tests do not include order as a factor.

We planned six comparisons. If a main effect of technique was found, we first compare Baseline results to each augmentation type. We also compare Mouse to Pocket, and Slowed to Blocked, to investigate the effect of level. Posthoc tests in subjective measures were corrected for multiple comparisons. Subjective results are reported per individual. Due to the ordinal nature of subjective ratings, we applied more-conservative non-parametric tests to these responses.

\section{RESULTS}

We first present the effects of our augmentations on crossing behaviour; we follow with how they influenced participants' subjective reports.

\section{Crossing Events}

We first wanted to determine whether the augmentations changed baseline behaviour. We ran a RM-ANOVA with the first Baseline and the four augmented embodiments (Slowed, Blocked, Mouse, and Pocket), and 4 planned contrasts (comparing each augmented embodiment with the baseline). There was a main effect of embodiment on the number of crossings $\left(F_{(4,60)}=13.3, p \approx 0.000, \eta^{2}=0.47\right)$.

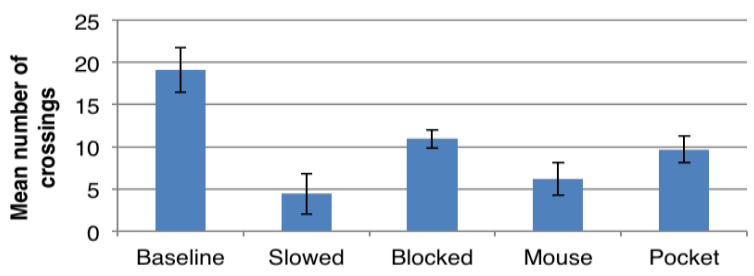

Figure 2 - Mean crossings $( \pm \mathrm{SE})$ by augmentation type.

Planned contrasts show that people crossed more with no augmentation (Baseline) than with all augmentation types (all $p<0.015$ ). See Figure 2.

We next wanted to determine whether the approach to augmentation (tactile feedback or movement alteration) and the level of augmentation (high or low) had an effect on crossing behaviour. We conducted a 2 (approach) $\times 2$ (level) RM-ANOVA on the number of crossings. There was no main effect of approach $\left(F_{(1,15)}=0.07, p=0.798, \eta^{2}=0.00\right)$, showing no difference between the tactile feedback approach and the movement alteration approach. However, there was a main effect of level $\left(F_{(1,15)}=10.0, p=0.006\right.$, $\left.\eta^{2}=0.40\right)$, showing that people crossed more with high level of augmentations than with the low level (see Figure 2).

\section{Permanence Effects}

We included a second baseline trial with an embodiment with no augmentations immediately after the first augmentation type. We did this to determine whether 
introducing an augmentation would have a lasting effect on crossing behaviour after the augmentation was removed. To answer this question, we conducted a RM-ANOVA with repetition of the two baselines as within-subjects data, and first augmentation type as a between-subjects factor. There was no main effect of repetition on crossing behaviour $\left(F_{(1,3)}=1.84, \quad p=0.200, \eta^{2}=0.13\right)$, and no interaction of repetition with starting condition $\left(F_{(3,12)}=0.69, p=0.578\right.$, $\left.\eta^{2}=0.15\right)$. Thus, people did not cross fewer times in the second baseline test, and this lack of difference was consistent across the four augmentations (see Figure 3). We interpret these results to mean there was no permanence effect: people resume behaving as they did before having experienced any augmentation.

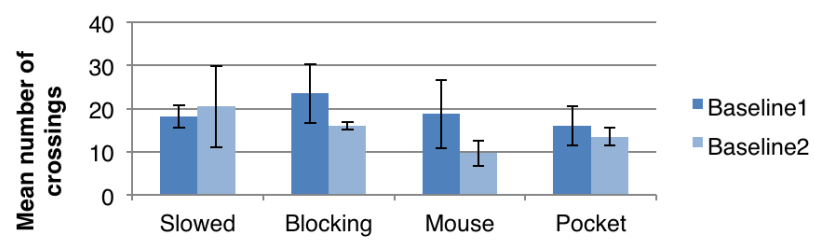

Figure 3 - Mean crossings $( \pm \mathrm{SE})$ between the Baseline trials

\section{Subjective Responses}

We asked participants eight questions to gather their perceptions of the augmentations. A RM-MANOVA with order as a between-subjects factor shows no effect of order for any of the subjective measures, except for awareness of partner's position $\left(F_{(3,25)}=4.4, p=0.012, \eta^{2}=0.347\right)$.

For each subjective response, we test for effects of augmentation type using a Friedman test. Pairwise comparisons (between Baseline and all conditions, between Mouse and Pocket, and between Slowed and Blocked) are investigated through Wilcoxon Signed Ranks Tests.

\section{Awareness}

People rated their agreement to the statement: I was aware of my partner's position on the table (see Figure 4). There was a main effect of augmentation type $\left(\chi_{(4)}^{2}=40.4, p \approx 0.000\right)$. Pairwise comparisons showed that awareness was lower for the Baseline than every augmentation type (Slowed $Z=-3.6$, Blocked $Z=-3.8$, Mouse $Z=-4.1$, Pocket $Z=-3.8$, all $p \approx 0.000)$. Blocked produced more awareness than Slowed $(Z=-2.3, p=0.020)$. There was no difference in awareness between the Mouse and Pocket augmentations $(Z=-0.7, p=0.498)$.

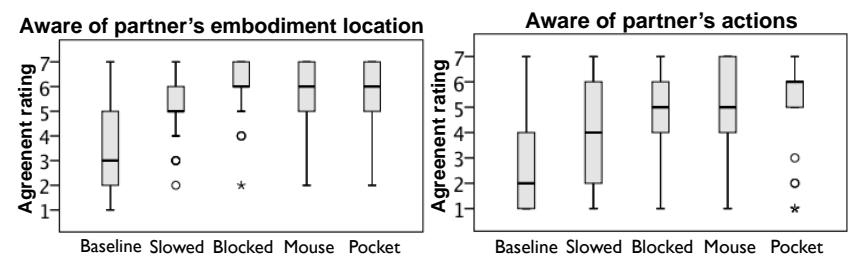

Figure 4 - Subjective ratings of awareness

People rated their agreement to the statement: I was aware of my partner's actions on the table (see Figure 4). There was a main effect of augmentation type $\left(\chi_{(4)}^{2}=33.0\right.$, $p \approx 0.000$ ). Pairwise comparisons showed that people were less aware in Baseline than every augmentation type (Slowed $Z=-2.4$, Blocked $Z=-2.8$, Mouse $Z=-3.2$, Pocket $Z=-3.2$, all $p<0.017)$. People were more aware when using Blocked than Slowed $(Z=-2.1, p=0.040)$. There was no difference between Mouse and Pocket $(Z=-0.4, p=0.725)$.

\section{Awkwardness}

People rated their agreement to the statement: It felt awkward to cross my partner's embodiment (see Figure 5). There was a main effect of augmentation type $\left(\chi_{(4)}^{2}=47.7\right.$, $p \approx 0.000$ ). Pairwise comparisons showed that people felt less awkward crossing in the Baseline condition than with every augmentation type (Slowed $Z=-3.9$, Blocked $Z=-3.9$, Mouse $Z=-4.1$, Pocket $Z=-4.2$, all $p \approx 0.000)$. People felt more awkward crossing embodiments when using Blocked than Slowed $(Z=-2.0, p=0.042)$. There was no difference between Mouse and Pocket $(Z=-1.3, p=0.178)$.

People rated their agreement to the statement: It felt awkward to reach to the other side of the table (see Figure $5)$. There was a main effect of augmentation $\left(\chi_{(4)}^{2}=30.0\right.$, $p \approx 0.000$ ). Pairwise comparisons showed that people felt less awkward reaching in the Baseline condition than with every augmentation type (Slowed $Z=-3.3$, Blocked $Z=-3.4$, Mouse $Z=-3.3$, Pocket $Z=-3.7$, all $p<0.001)$. There was no difference between Blocked and Slowed $(Z=-1.9, p=0.056)$ or between Mouse and Pocket $(Z=-1.6, p=0.104)$.
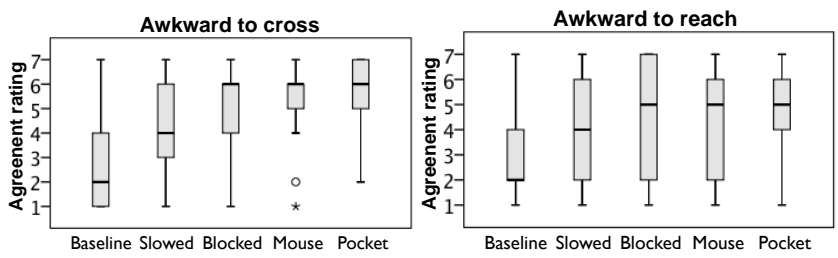

Figure 5 - Subjective ratings of awkwardness

\section{Feelings of Invasion}

People rated their agreement to the statement: I felt like my partner was invading my space (see Figure 6). There was a main effect of augmentation type $\left(\chi_{(4)}^{2}=29.1, p \approx 0.000\right)$. Pairwise comparisons showed that people felt less invaded in the Baseline condition than in every augmentation type (Slowed $Z=-2.9$, Blocked $Z=-3.3$, Mouse $Z=-3.2$, Pocket $Z=-3.4$, all $p<0.004)$. People felt more invaded with Blocked than with Slowed $(Z=-2.2, p=0.028)$, and more invaded with Pocket than with Mouse $(Z=-2.3, p=0.024)$.

People rated their agreement to the statement: I felt like I was invading my partner's space (see Figure 6). There was a main effect of augmentation type $\left(\chi_{(4)}^{2}=38.9, p \approx 0.000\right)$. Pairwise comparisons showed that people felt less invading in the Baseline condition than in every augmentation type (Slowed $Z=-3.1$, Blocked $Z=-3.1$, Mouse $Z=-3.5$, Pocket $Z=-3.8$, all $p<0.002)$. People also felt more invading with Blocked than Slowed $(Z=-2.0, p=0.043)$, and more invading with Pocket than Mouse $(Z=-2.4, p=0.017)$. 


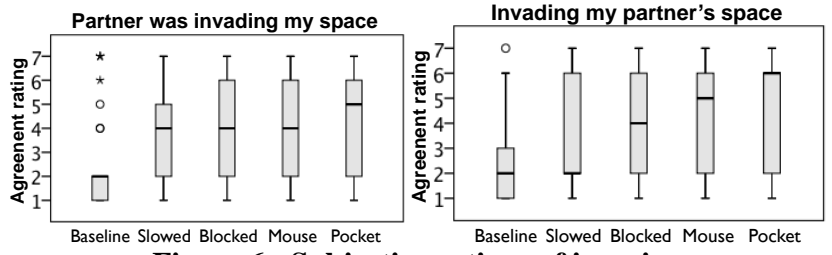

Figure 6 - Subjective ratings of invasion

\section{Frustration and Annoyance}

People rated their agreement to the statement: This embodiment was annoying to use (see Figure 7). There was a main effect of augmentation type $\left(\chi_{(4)}^{2}=36.1, p \approx 0.000\right)$. Pairwise comparisons showed that it was less annoying in the Baseline condition than in every augmentation type (Slowed $Z=-3.3$, Blocked $Z=-3.8$, Mouse $Z=-3.8$, Pocket $Z=-3.9$, all $p<0.001)$. There was no difference between Blocked and Slowed $(Z=-1.3, p=0.185)$, or between Mouse and Pocket $(Z=-1.2, p=0.212)$.

People rated their agreement to the statement: It was frustrating to coordinate with my partner to avoid touching (Figure 7). There was a main effect of augmentation type $\left(\chi_{(4)}^{2}=40.4, p \approx 0.000\right)$. Pairwise comparisons showed that it was less frustrating in the Baseline condition than in every augmentation type (Slowed $Z=-3.0$, Blocked $Z=-3.1$, Mouse $Z=-3.7$, Pocket $Z=-3.6$, all $p<0.003)$. There was no difference between Blocked and Slowed $(Z=-1.3, p=0.195)$, or between Mouse and Pocket $(Z=0.0, p=1.000)$.
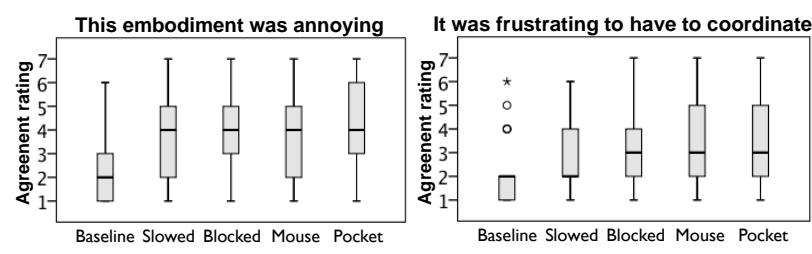

Figure 7 - Subjective ratings of annoyance and frustration

\section{Interview Responses}

Frustration: 12 groups explicitly stated that it was not frustrating to have to coordinate with the other person. 4 stated it may be frustrating if there was a time limit, evaluation of haiku quality, or if they were strangers.

Cell phone: 3 groups stated that Pocket was like a cell phone in their pocket, a feeling they are accustomed to. 1 stated the Mouse was like a game controller.

Mouse noise: 7 groups stated that the loudness of the Mouse vibration increased the disruption of the Mouse.

Slowed: 9 groups reported that Slowed was frustrating and annoying because it was slow to recover from the cross. Most preferred Blocked, because it prevents crossing, whereas Slowed suggests you still can, but punishes you.

\section{Observed Episodes}

We observed clear behaviour change and an increase in coordinative, turn-taking behaviour. The following episodes describe situations observed during the study.
Scanning. Many people moved their embodiments around the table as a pointing aid while searching for words. This is common behaviour with physical arms (e.g., while reading a book), but rarely occurs on physical tabletops. We observed scanning behaviour change with augmentations. Most people stopped scanning, and kept their cursors near to their haiku to avoid crossing; however, this was not true for all groups. One group (211) scanned during the entire experiment, causing many crossings in all haiku tasks.

Alternating. Many groups quickly formed a turn-taking strategy. A common strategy was alternating, where each person takes one turn in quick succession. Alternating behaviour is a clear and effective turn-taking technique that requires good awareness of the other's actions. An obvious instance of alternating behaviour was from group 9. Person $\mathbf{A}$ (Figure 1) has her embodiment on her haiku. $\mathbf{B}$ reaches in front of A's haiku and grabs a word. B waits until A moves out of the way, and then reaches in front of A's haiku. B waits her turn, and then reaches to the other side of the table. This alternating continues until each person picked three words each in quick succession. This occurred with Blocked, which forces groups to take turns; however, not all groups had good alternating behaviour, reaching when the other person was in the way, and causing a collision.

False starts. As part of turn-taking, we observed numerous false starts - when one person is in the way, the second person begins to move but realizes there will be a collision, and so stops and waits. False starts are a clear indication that people had good awareness of the other person's embodiment location, because they were able to prevent the collision from happening. A good example of a false start was in group 6. B is in his haiku. A reaches to the top of B's side. B looks to the other side of the table and starts to move, but sees that $\mathbf{A}$ is in the way. He pauses, and waits. $\mathbf{A}$ tries to grab a word, and begins to move her embodiment, but missed the word, so reaches forward again. B waits and watches her embodiment as she selects the word. When she pulls back, $\mathbf{B}$ grabs a word that was under her embodiment.

\section{DISCUSSION}

Our study shows that both tactile feedback and movement alteration can change interaction in tabletop tasks, can make people more aware of another person's digital embodiment, and can make people more sensitive to feelings of intrusion and awkwardness. These changes were also accompanied, however, by increased feelings of annoyance and frustration at having to coordinate with the augmented embodiments. In the following sections, we provide explanations for these main results, and consider how our findings can be used by researchers and designers of tabletop systems.

\section{Interpretation of Main Results}

Our primary result is that when tactile feedback and movement alteration were present, people's behaviour with, and perception of, the digital embodiments changed 
substantially. The next paragraphs summarize and explain the effects for each of the augmentations in turn.

Slowed Movement. People crossed least with the Slowed embodiments overall, and significantly less often than with the Blocked embodiment. This latter result is the opposite of what we expected, because Blocked incurs a larger movement penalty than Slowed. However, interview results suggest that this was not how groups interpreted these augmentations. Groups reported that Slowed punished performance in both directions; that is, it required that people move slowly to perform a crossing action, and also move slowly to recover from the cross. In contrast, even though Blocked was more restrictive, it did not reduce the local responsiveness of the embodiment. This difference seems to have caused greater interaction avoidance than with any other embodiment.

Blocked Movement. People crossed more with Blocked than with Slowed, but reported being more aware with Blocked, and reported that Blocked was more invasive. These results seem contradictory: if Blocked is more invasive and causes better awareness, why did people cross more? We see three reasons. First, people knew they had to change their behaviour, because Blocked completely prevents people from crossing; however, there was no interaction penalty to recover from the cross (unlike Slowed), so people felt free to collide or bump into the other Blocked embodiment. Second, people often poked at the other person's embodiment to signal them to move out of the way, leading to an increased number of crossing events (even though these were not intended as crosses). Third, the lack of a real performance cost may have caused people to be sloppier with their actions, preferring to simply interact and then recover (which was quick and easy) from collisions.

Mouse Tactile Feedback. People crossed less with Mouse than with Pocket, and reported Mouse as less invasive than Pocket. The crossing result is again the opposite of what we expected, but the invasiveness result matches expectations. On the intimacy scale, a touch on the thigh is much more intimate than a touch on the hand; however, people crossed less with tactile feedback on their hands than on their thighs. It appears that the reduction in crossing was not due to the increased intimacy of the location. Instead, people reported that it was the increased perceptual intrusiveness of the Mouse that caused them to avoid crossing. The Mouse vibration was more obvious than the Pocket vibration, partly because it was louder (i.e., the mouse buzzed against the table); as a result, people reported that it was more distracting and it broke their concentration.

Pocket Tactile Feedback. People crossed more with Pocket than Mouse, and reported Pocket to be more invasive than Mouse. The subjective results show that the intimacy scale on which we based the tactile augmentations was correct, and that tactile sensations and body location do have some governing effect on behaviour. However, the results suggest that pocket vibrations are not overly intimate for some people. Several participants reported that this condition "felt like a cell phone", although others stated that it was uncomfortable. This suggests there is a familiarity effect: people who are used to having a cell phone vibrate in their pocket interpreted this tactile feeling as less invasive than those that are not accustomed to the feeling.

\section{Using the Findings in Tabletop Design}

Our study showed that even in the absence of true physicality, designers of tabletop groupware can find ways of influencing and shaping interaction and awareness. An obvious question, however, that arises from these findings is whether, and when, it is useful for designers to add constraints to interaction. We address several issues that surround this question, including the design goal of the augmentation, the tension between discomfort and utility, and the use of public actions as a basis for social protocols.

\section{The design goal of adding artificial constraints}

First, it is important to note that the goal of our exploration is not to simply try and replicate the constraints of the physical world - i.e., to duplicate the behaviours that arise with physical arms and hands. It is clear that this did not happen in our study: even though interaction changed, it was clear that the behaviour was different in many ways from what has been observed with physical arms in past work [8]. For example, people reacted to the obviousness and interruption of the tactile feedback as much as they did to the intimacy of the feedback; similarly, the movementbased manipulations led to people calculating the penalty to their own work rather than recreating a notion of touch.

There are two goals in adding artificial augmentations to embodiment interaction: first, to better understand what governs and shapes shared behaviours like awareness, coordination, and territoriality; and second, to make use of those factors to increase the range of experiences that designers can provide in a tabletop groupware system. We hypothesize a 'continuum of control' in group interactions, where at one end there are no constraints on behaviour, and at the other end there are rigid structures and regulations that affect people's every move. In previous CSCW research, these structures have often been built into the task interface itself (e.g., floor control); in this research we are instead exploring implicit forms of control that arise from characteristics of embodiments, awareness, and interaction.

In the space of tabletop systems, there are several valuable points along the continuum of control. There are situations toward the 'unconstrained' end of the spectrum where the lack of awkwardness in interactions may be beneficial. For example, in time-critical systems, users can work faster knowing their interactions will not inconvenience others. There are other situations (e.g., safety-critical applications) that demand that coordination conflicts be minimized. Systems to support these situations could benefit from the addition of artificial (but still implicit) constraints. 
Tension between discomfort and usefulness

Causing discomfort for people around the table - that is, adding factors that increase awkwardness and intrusion, and that increase people's annoyance and frustration as a result - may seem like a strange design strategy. However, recent research in $\mathrm{CHI}$ and $\mathrm{CSCW}$ has shown that there are legitimate reasons to use discomfort as a design principle: for example, Benford et al. suggest that 'uncomfortable interactions' can provide potential benefits in several areas such as entertainment, enlightenment, and sociality [4]. In the case of tabletop interaction, it is clear that discomfort underlying touch avoidance leads to obvious benefits for the group in terms of their ability to operate successfully and smoothly in a constrained space.

As previous researchers have noted, there are always tradeoffs between designing for the individual and designing for the group in shared-workspace systems [10]. It may be that the uncomfortable interaction of close physical contact is a fundamental part of people's natural coordination abilities around tables; therefore, it is not so strange to expect increases in individual feelings of awkwardness or annoyance when attempting to improve group awareness. In addition, in situations like the safetycritical scenario mentioned above, group members may be willing to give up some degree of individual control in order to have a better sense of the group's location and activity, and to reduce errors and conflicts.

\section{Making actions public}

One of the main properties of both augmentation types studied here is that they make embodiment interactions much more obvious - when crossings occurred, people received tactile, auditory, and movement-based feedback. The obviousness of this feedback can play a role in the development of rules for social behaviour. For example, people may have been more reluctant to cross with the noisy Mouse vibration because the feedback was clearly obvious to both parties; similarly, the Slowed condition was an effect that was particularly public (in that it slowed down both people, not just the person crossing).

Several researchers have noted that when actions are public, people change their behaviour (for example, people are much less likely to watch another person if the watching behaviour is made public) [5]. We are interested to see how social protocols may evolve around different kinds of public signals that are produced when embodiments interact - we wonder whether the obviousness of the signal will provide a stronger impetus to form new social protocols.

\section{Generalization and Limitations}

Our study involved a large sample and well-controlled conditions, so we are confident that our effects can be replicated for two-person work in other tasks and scenarios. However, there are also limitations to the work. We did not collect an objective measure of awareness; instead, we collected crossing events, which we use as a proxy for awareness (i.e., you need to know where the other person is to avoid touching them). Second, we do not know how the augmented embodiments would scale to larger groups. As the number of embodiments increase, there are more opportunities for conflicts, meaning that people may be constantly receiving feedback (and thus start to ignore it). However, we note that in the physical world, we are still able to avoid touching others even when there are more than two people interacting over the table.

\section{CONCLUSION AND FUTURE WORK}

Digital embodiments support coordination and awareness far less well than physical arms, partly due to the loss of touch avoidance that occurs naturally in the physical world. There is little understanding of other factors that may guide and govern tabletop interactions, leaving designers with few options as they attempt to provide a wide range of collaborative tabletop experiences. To add to this understanding, we carried out a study that demonstrated the effectiveness of tactile feedback and movement alteration in changing behaviour and improving group awareness. Our work provides designers with new understanding of group interaction, and provides tools and strategies for creating richer and more complex behaviour in tabletop groupware.

In future work, we plan to explore these findings in more detail. We will address the limitations noted above (objective measures of awareness and coordination, and studies with larger groups). In addition, we are interested in replicating our effects in distributed settings: both for networked tables, and possibly even for standard desktopbased groupware. Finally, we are interested in studying the possibility that new social protocols may develop over time that adapt to the new artificial constraints.

\section{ACKNOWLEDGEMENTS}

We thank NSERC, Surfnet, and the Walter C. Sumner fellowship for funding, the HCI lab at the $U$ of $S$ for feedback, and all of our participants.

\section{REFERENCES}

1. Anderson, P.A., Leibowitz, K. The Development and Nature of the Construct Touch Avoidance. Nonverbal Behaviour. 3(2). 1978. 89-106.

2. Back, J., Brumby, D. P., and Cox, A. L. Locked-out: Investigating the Effectiveness of System Lockouts to Reduce Errors in Routine Tasks. CHI 2010. 3775-3780.

3. Bailensen, J. N., Yee, N., Brave, S., Merget, D., and Koslow, D. Virtual Interpersonal Touch: Expressing and Recognizing Emotions through Haptic Devices. HumanComputer Interaction. 2007. 325-353.

4. Benford, S., Greenhalgh, C., Giannachi, G., Walker, B., Marshall, J., and Rodden, T. Uncomfortable Interactions. CHI 2012. 2005-2014.

5. Birnholtz, J., Gutwin, C., Hawkey, K. Privacy in the Open: How Attention Mediates Awareness and Privacy in Open-Plan Offices. Group 2007, 51-60. 
6. Cockburn, A., Kristensson, P. O., Alexander, J., and Zhai, S. Hard Lessons: Effort-Inducing Interfaces Benefit Spatial Learning. CHI 2007. 1571-1580.

7. de Korte, E.M., de Kraker, H., Bongers, P.M., and van Lingen, P. Effects of a feedback signal in a computer mouse on movement behaviour, muscle load, productivity, comfort and user friendliness. Ergonomics. 51(11). 1157-1775.

8. Doucette, A., Gutwin, C., Mandryk, R.L., Nacenta, M., Sharma, S. Sometimes when we touch: How Arm Embodiments Change Reaching and Coordination on Digital Tables. CSCW 2013.

9. Greenberg, S. and Marwood, D. Real time groupware as a distributed system: concurrency control and its effect on the interface. CSCW 1994. 207-217.

10. Gutwin, C. and Greenberg, S. Design for individuals, design for groups: tradeoffs between power and workspace awareness. CSCW'98, 207-216.

11.Ha, V., Inkpen, K.M., Mandryk, R.L., Whalen, T. Direct Intentions: The Effects of Input Devices on Collaboration around a Tabletop Display. TABLETOP 2006. 177-184.

12. Haans, A. and IJsselsteijn, W. Mediated social touch: a review of current research and future directions. $V R$ 2006. 149-159.

13. Hall, E.T. The Hidden Dimension. 1966.

14. Heslin, R, Nguyen, T.D., Nguyen, M.L. Meaning of touch: The case of touch from a stranger or same sex person. Nonverbal Behaviour. 7(3). 1983. 147-157.

15.Hornecker, E., Marshall, P., Dalton, N.S., Rogers, Y. Collaboration and interference: awareness with mice or touch input. $C S C W 2008$. 167-176.

16. Hullman, J., Adar, E., Shah, P. Benefiting InfoVis with Visual Difficulties. IEEE Trans. Vis. and Graphics. 17(12). 2213-2222.

17.Izadi, S., Brignull, H., Rodden, T., Rogers, Y., Underwood, M., Dynamo: A public interactive surface supporting the cooperative sharing and exchange of media. UIST '03. 159-168

18. Jeffrey, P., Mark, G. Navigating the virtual landscape: co-ordinating the shared use of space. In Social Navigation of Information Space, 112-131.

19. Jourard, S.M. An Exploratory Study of BodyAccessibility. J. Soc. Clin. Psych., 5, 1966. 221-231.

20. Kruger, R., Carpendale, S, Scott, S.D., and Greenberg, S. How people use orientation on tables: comprehension, coordination and communication. GROUP 2003. 369-378.

21. Mandryk, R. and Gutwin, C. Perceptibility and Utility of StickyTargets. GI 2008, 65-72.

22. Morris, M.R., Cassanego, A., Paepcke, A. Winograd, T., Piper, A.M., and Huang, A. Mediating Group Dynamics through Tabletop Interface Design. 2006. IEEE Computer Graphics and Applications 26(5), 65 -73
23. Morris, M., Ryall, K., Shen, C., Forlines, C., Vernier, F. Beyond "social protocols": multi-user coordination policies for co-located groupware. CSCW'04. 262-265.

24. Nacenta, M.A., Pinelle, D., Gutwin, C., Mandryk, R.L. Individual and Group Support in Tabletop Interaction Techniques. TABLETOP 2010, 303-333.

25. Nacenta, M.A., Pinelle, D., Stuckel, D., Gutwin, C. The effects of interaction technique on coordination in tabletop groupware. GI 2007. 191-198.

26. Nguyen, T., Heslin, R., Nguyen, M.L. The meanings of touch: Sex differences. Comm. '73. 25(3). 92-103.

27. O'Hara, K. P. and Payne, S. J. Planning and the user interface: the effects of lockout time and error recovery cost. Int. Jour. Hum.-Comp. Studies. 1999. 50(1). 41-59.

28. Oakley, I., Brewster, S., and Gray, P. Communicating with Feeling. Haptic HCI 2001. 61-68.

29. Owen, J.M., Petro, J.A., D'Souza, S.M., Rastogi, R., and Pawluk, D.T.V. An improved, low-cost tactile 'mouse' for use by individuals who are blind and visually impaired. ASSETS 2009. 223-224.

30. Pinelle, D., Barjawi, M., Nacenta, M., and Mandryk. R.L. An evaluation of coordination techniques for protecting objects and territories in tabletop groupware. CHI 2009. 2129-2138.

31.Pinelle, D., Nacenta, M., Gutwin, C., Stach, T. The effects of co-present embodiments on awareness and collaboration in tabletop groupware. GI 2008. 1-8.

32.Scott, S.D., Carpendale, S.T., Inkpen, K.M. Territoriality in collaborative tabletop workspaces. CSCW 2004. 294-303.

33. Slater, M., Steed, A. Meeting People Virtually: Experiments in Shared Virtual Environments. The Social Life of Avatars: Presence and Interaction in Shared Virtual Environments. 2002.

34. Smith, R.B., Hixon, R., Horan, B. Supporting flexible roles in a shared workspace. CSCW 1998. 197-206.

35. Tang, A., Pahud, M., Inkpen, K., Benko, H., Tang, J.C., Buxton, B. Three's Company: Understanding Communication Channels in Three-way Distributed Collaboration. CSCW2010. 271-280.

36. Tang, A., Neustaedter, C., Greenberg, S. VideoArms: Embodiments for Mixed Presence Groupware. People and Computers, in Proc of HCI '06. 2-18.

37. Thayer, S. History and Strategies of Research on Social Touch. Nonverbal Behaviour. 10(1). 1986. 12-28.

38. Tsandilas, T. and Balakrishnan, R. An Evaluation of Techniques for Reducing Spatial Interference in Single Display Groupware. ECSCW 2005. 225-245.

39. Tse, E., Histon, J., Scott, S.D., Greenberg, S. Avoiding interference: how people use spatial separation and partitioning in SDG workspaces. CSCW 2004. 252-261.

40. Vanacken, D., Raymaekers, C., Luyten, K., Coninx, K. Focus+Roles: Socio-Organizational Conflict Resolution in Collaborative User Interfaces. HCI 2007. 788-796. 
